\title{
The breast cancer conundrum
}

For decades, rates of breast cancer have been going up faster in rich countries than in poor ones. Scientists are beginning to understand more about its causes but unanswered questions remain. Patrick Adams reports.

Nearly 40 years ago, former first lady of the United States of America (USA), Betty Ford, announced to the world that she had been diagnosed with breast cancer and would undergo a radical mastectomy to remove the tumour. Ford's brave decision to make her diagnosis public broke the silence around the disease and prompted millions of women to go for screening. And, as they did, detection rates in the USA rose sharply. Researchers called it the "Betty Ford blip."

By contrast, the increasing rates of breast cancer observed in the United States and other affluent countries over the past three decades cannot be explained by greater awareness and screening alone. And, while mutations to the genes BRCA1 and BRCA2 have been shown to dramatically raise a woman's lifetime risk of breast cancer, fewer than $10 \%$ of cases are thought to occur in women with these hereditary mutations.

It remains to be seen whether other forms of hereditary breast cancer exist. Most breast cancer cases occur in women with no known inherited risk factors, leading scientists to wonder what else is going on.

What is it about women's lifestyles in developed countries that makes them so much more likely to develop the disease than their counterparts in parts

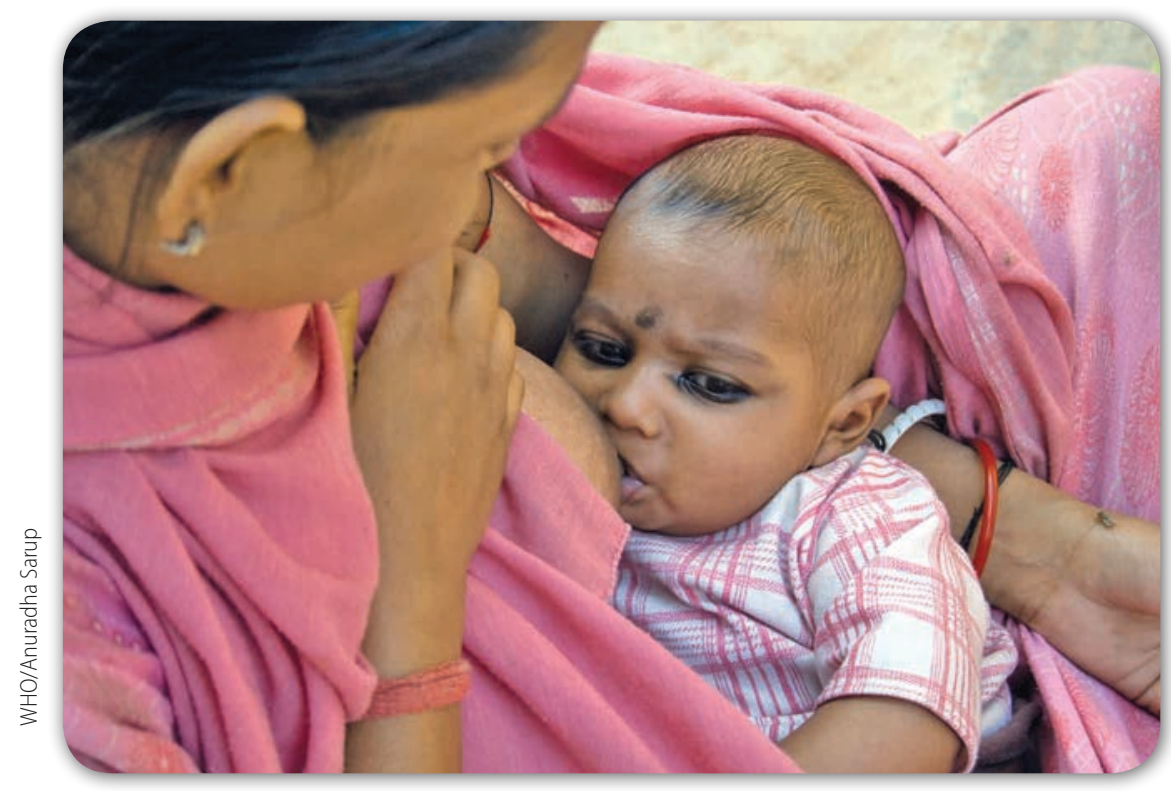

Breastfeeding is seen as a potentially protective factor against breast cancer of south-east Asia and Africa, where incidence is typically five times lower?

Can the discrepancy be explained, in part, by low detection rates for breast cancer in poor countries, where the real able data suggest?

"It's not that some populations are genetically immune to the disease," and cancer expert at the University of Oxford, "because we know that when people from poor countries move to rich countries, within one or two generations they have the same rates as Westerners. It's something about the way people live."

Obesity and alcohol consumption are clearly involved, says Key. "Women who are obese have higher blood levels of [the hormone] oestrogen and that's because fat cells make oestrogen," which fuels the growth of most breast cancer tumours. Alcohol, too, is associated with higher blood levels of hormones, which may be the mechanism by which it increases a woman's breast cancer risk. And several recent studies show that physical activity can reduce risk, by contributing to weight control and by additional mechanisms.

In terms of lifestyle, childbearing may reveal the most about the global variation in breast cancer risk. "You get low rates in parts of Africa because burden may be much higher than availsays Dr Tim Key, an epidemiologist

women start having children when they're young, have several children, and breastfeed them for a long time," says Key. The lower rates may be due, in part, to structural changes in breast tissue and a reduction in the number of stem cells, he says. But it's also likely that childbearing lowers a woman's risk by reducing her exposure to oestrogen.

We know that
when people from
poor countries move to
rich countries, within
one or two generations
they have the same
rates as Westerners. It's
something about the
way people live.
Tim Key

"The more menstrual cycles a woman has over her lifetime, the greater is her risk of breast cancer," says Dr Philip Landrigan, a paediatrician and epidemiologist at Mount Sinai Medical Center in New York and a leading expert on the effects of exposure to environmental hazards. "Every time a woman gets pregnant, that's nine or 10 cycles that she doesn't have. And since we know that women who enter puberty earlier are going to have more cycles, we're very concerned about the fact that girls are going into puberty one to two years earlier than they were a generation ago."

For that, Landrigan adds, diet may deserve some of the blame; studies have shown that girls who are overweight or obese tend to go into puberty earlier.

Hormone replacement therapy and oral contraceptives, both sources of oestrogen, have also been shown to be risk factors for breast cancer.

Landrigan and other scientists are particularly concerned about chemicals called endocrine disrupting compounds (EDCs). Found in many things, from deodorants, sunscreens, cosmetics and food packaging materials to pesticides and dental appliances, EDCs have been shown to mimic, magnify, alter and 
even block the effects of oestrogen, which, among other things, regulates the sequence and timing of breast development.

Controversy has long surrounded the effect of EDCs on human health, ever since the environmental devastation wrought by DDT became evident 50 years ago and the pesticide was banned in many countries. But, with regard to DDT's effect on the risk of breast cancer, the epidemiological data are mixed.

"This is one of the challenges in the broader field of carcinogen identification," says Dr Kurt Straif, an epidemiologist with the World Health Organization's (WHO) International Agency for Research on Cancer (IARC) and head of its long-running monograph series on the evaluation of carcinogenic risks to humans, the world's most comprehensive catalogue of its kind.

"What's usually considered the strongest evidence that something is causing cancer in humans is epidemiological studies showing a causal association between an exposure in humans and cancer in humans. Considering the long latency period for some cancers, it could well be that there is no evidence of an increase in cancer risk in the first 20 years after first exposure." And indeed, most of the 30 or so studies of DDT and breast cancer risk - be they case-control comparisons of surgical tissues, prospective studies with follow-up duration of less than 14 years, or retrospective studies that

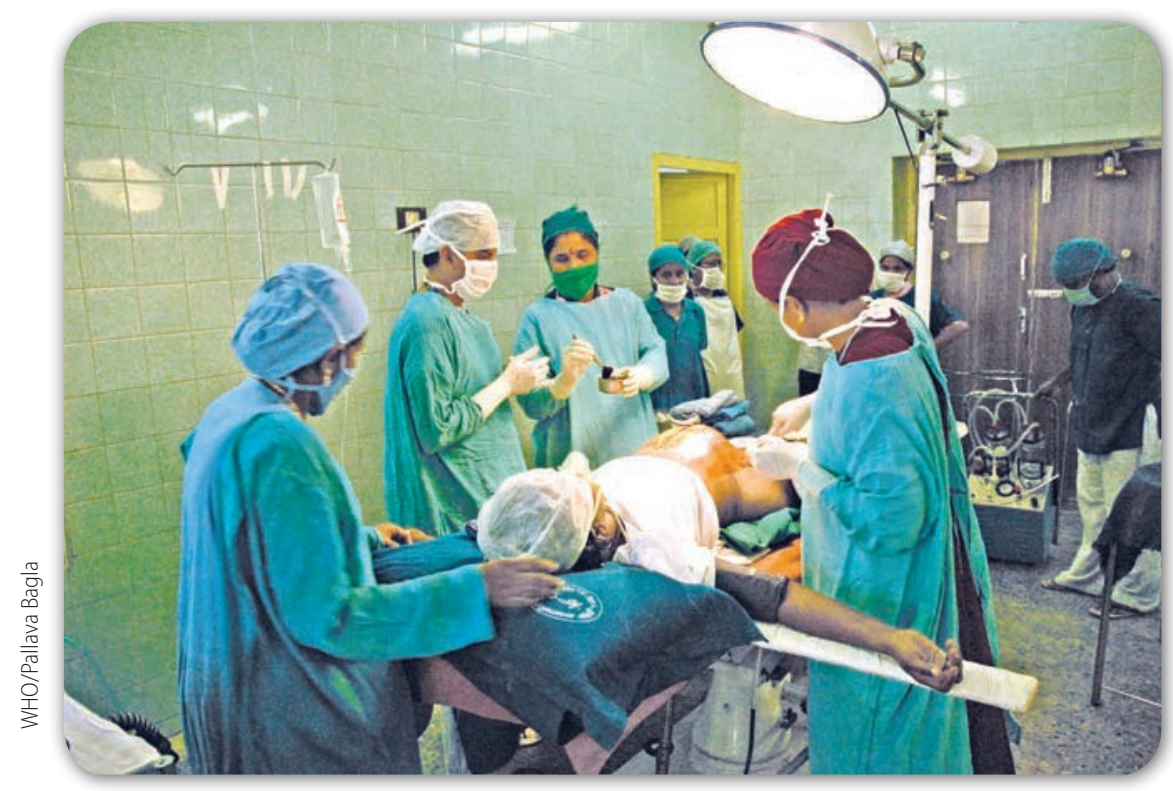

Shift work that disrupts normal sleep routines could be a factor for developing breast cancer rely on serum samples collected from women in middle age or later - share that important limitation.

In 2007, Dr Barbara Cohn and colleagues at the Public Health Institute's Child Health and Development Study project published the findings of a study using blood samples of young women collected between 1959 and 1967, at the peak of DDT use in the United States. It found that exposure to DDT during childhood and early adolescence was associated with a fivefold increase in risk of developing breast cancer before the age of 50 .

"This is really important," says Landrigan, whose own work on children's exposure to lead in the United States formed the scientific basis for the federal ban on lead paint in the 1970s and its eventual elimination from gasoline. "It's a landmark work. We know from animal toxicology studies that many different chemicals increase the risk of breast cancer in animals. But this is one of the first really good demonstrations of that in humans."

A WHO expert panel reviewed several studies, including the one by Cohn and colleagues, and concluded in its 2011 report, DDT in indoor residual spraying: human health aspects, that DDT exposure was "generally not associated with breast cancer". "There are some positive studies, but they are outweighed by an overwhelming number of negative studies," it found.

None of the studies were conducted in malaria-endemic parts of sub-Saha- ran Africa and Asia, where DDT spraying has been widely practised to kill the mosquito vector.

"There is stronger evidence for shift work involving circadian disruption as a factor for developing breast cancer than for chemicals," Straif of IARC says.

Shift work involving disruption of normal sleep routines is currently classified as an "IARC 2A carcinogen," he adds, which means that it is probably carcinogenic to humans.

\section{Most funding is going toward finding a cure. 7 \\ Janet Gray}

This linkage was reinforced by studies published recently that further support a causal association, he says: "Given the prevalence of shift work in modern societies, this could be an important risk factor on the population level."

According to Key of Oxford University: "Chemicals such as DDT might be important but this is not established. More research is needed. For women who want to reduce their own risk, the things they can control that will definitely make a difference are body weight and alcohol consumption."

Nevertheless, the Breast Cancer Fund and other activists are calling for a precautionary approach to chemical regulation in the United States and argue that not enough is spent on research to find ways to prevent breast cancer.

"Most funding is going toward finding a cure," says Dr Janet Gray, lead author of the 2008 and 2010 editions of State of the evidence, a comprehensive report released by the San Franciscobased advocacy group, the Breast Cancer Fund.

"I want to find a cure. But I would rather prevent the disease, when that is possible," Gray says, adding: "It is absolutely critical that we begin to think about links between exposures to environmental chemicals and disease as a public health issue. The goal should be to decrease exposures and prevent the development of diseases." 